\title{
Mechanical Considerations of Bioprinted Tissue
}

\author{
Hannah A. Strobel, Sarah M. Moss and James B. Hoying * \\ Advanced Solutions Life Sciences, Manchester, NH, United States
}

Bioprinting enables the fabrication of engineered tissues with complex features and is conducive to automated tissue manufacturing and scale up. As cell systems and tissues are dynamic and very responsive to environmental cues, including biomechanical stimuli, the form and function of bioprinted tissues can change dramatically after printing. Here, we discuss tissue biomechanics relevant to printed cell systems and tissues emphasizing the dynamic and complex relationship between cell behavior, matrix deposition, and tissue forces. Furthermore, we provide perspectives on the control and manipulation of mechanical forces and designed boundary conditions for tissue constructs in printing-based approaches and strategies.

\section{OPEN ACCESS}

Edited by:

Bahattin Koc,

Sabancı University, Turkey

Reviewed by:

Subhadip Bodhak,

Central Glass and Ceramic Research

Institute (CSIR), India

Srikanth Bontha,

National Institute of Technology,

Karnataka, India

Sourabh Ghosh,

Indian Institute of Technology Delhi,

India

${ }^{*}$ Correspondence:

James B. Hoying

jhoying@advancedsolutions.com

Specialty section: This article was submitted to Digital Manufacturing,

a section of the journal

Frontiers in Mechanical Engineering

Received: 02 June 2020 Accepted: 05 October 2020 Published: 28 October 2020

Citation:

Strobel HA, Moss SM and Hoying JB (2020) Mechanical Considerations of

Bioprinted Tissue.

Front. Mech. Eng. 6:568726.

doi: $10.3389 /$ fmech.2020.568726
Keywords: bioprinting, tissue mechanics, bioink, mechanical testing, tissue fabrication

\section{INTRODUCTION}

There are many considerations when designing a tissue, including shape, cell composition, matrix elements, and any needed maturation processes. The complex interplay between the varied components, including mechanical dynamics, can greatly influence the form and function of a fabricated tissue. Bioprinting is a rapidly progressing field, enabling the fabrication of a variety of biomedical-related constructs and structures, including tissue therapeutics (Datta et al., 2017; Xiang et al., 2020), in vitro tissue models and phantoms (Mao et al., 2020; Mota et al., 2020; Sasikumar et al., 2020), and biomedical devices (Bedell et al., 2020). Furthermore, bioprinting, as a type of additive manufacturing, is amenable to scaled up tissue fabrication and manufacturing, an important consideration as clinical use of fabricated tissues increases (Wu et al., 2017; Skylar-Scott et al., 2019; Castilho et al., 2020). The development of materials compatible with cells and other biologics, so called "bioinks" has also exploded involving a variety of artificial and native material types (Chimene et al., 2016; Chan et al., 2020; Cui et al., 2020), material sourcing (Abaci and Guvendiren, 2020), curing features (GhavamiNejad et al., 2020), and utilities (Whitford and Hoying, 2016; Unagolla and Jayasuriya, 2020). While the practical aspects and applications involving bioprinting are actively explored (as indicated by the recent burst of informative reviews on bioprinting and bioinks), the integration of biological responses and dynamics in bioprinted systems are less addressed. Given that biological systems are very attuned to the environmental cues, including those created via bioprinting, considerations as to how these different cues, such as with biomechanical forces, are critical in producing appropriate tissues and tissue constructs. In this mini review, we discuss the importance of tissue mechanical properties and the associated challenges when manufacturing a bioprinted tissue.

\section{“4D” BIOPRINTING OF TISSUES}

Conceptually, 3D printing of biological systems is in practice a " $4 \mathrm{D}$ " process, with dynamic responses and adaptations occurring (the fourth aspect) after printing of the 3D structure (Whitford and Hoying, 2016). In the context of materials and biomaterials science, $4 \mathrm{D}$ typically refers to changes in form and 
function of a printed object due to intrinsic, often self-actuating, features used in the print (Unagolla and Jayasuriya, 2020). For example, printed materials that swell in the presence of an additive or deform secondary to intramolecular interactions following exposure to light (Clegg et al., 2019). The additional functionalities of these so called "smart materials" contribute to programmed and anticipated changes in shape beyond the initial printed form.

Translating this $4 \mathrm{D}$ concept to biological systems, cellular systems also undergo spontaneous, self-actuated activities following printing, such as proliferation, differentiation, matrix deposition, and organization. Biomolecules and cells are constantly responding to environmental cues including those derived from biochemical signals, cell:cell communications, and biomechanical forces. Biomechanical cues manifest in a variety of ways such as contact guidance by the extracellular matrix, internal stresses generated by the cells themselves, external forces applied to the tissue environment, and boundary condition constraints. Rarely will a 3D printed tissue or tissue model remain as it was when printed (Figure 1).

All of these cues can influence the outcome of a fabricated tissue construct. While these post-printing dynamics can frustrate efforts to derive a tissue with a specific form and function, they can also be leveraged as part of a fabrication strategy to produce desired outcomes that might otherwise be difficult to pre-ordain. For example, when angiogenic vessel segments are printed in parallel arrangements using $3 \mathrm{D}$ printing, the microvasculature that formed after implantation did not keep this pre-patterned topology (Chang et al., 2012). However, 3D printing the microvessel segments while simultaneously constraining boundary conditions favorable to vessel alignment during remodeling did produce the desired topology due to secondary effects of matrix strain on the microvessels (Chang et al., 2012; Underwood et al., 2014). Here, leveraging external mechanical cues was essential for creating the desired tissue properties, more so than initial placement of vessel segments during fabrication. Similarly, leveraging the intrinsic contractile capabilities of fibroblasts produces thin, tissue fibers with high tensile strengths that would otherwise be difficult to immediately derive ( $\mathrm{Li}$ et al., 2016; Nakanishi et al., 2019). In virtually all cases, and highlighted by these examples, the dynamic interplay between the cells, the ECM (whether native or artificial), and the boundary conditions of the tissue construct profoundly influence outcomes and, consequently, initial design and tissue printing strategies.

\section{THE TISSUE ENVIRONMENT: ECM}

Often intimately associated with the cellular elements of a tissue, the ECM is an essential component to the tissue environment. Comprising a complex network of diverse biomolecules, the ECM provides a variety of physical and biochemical cues to cells determining cell phenotype and, ultimately, tissue function (Discher et al., 2009; Murphy et al., 2014; Arteel and Naba, 2020; Espana and Birk, 2020). Furthermore, the ECM establishes much of the mechanical properties of tissues, alone or in combination with cells, as well as influences cell behaviors contributing to the active mechanical aspects of each tissue (Arteel and Naba, 2020). For example, the high mechanical strength and viscoelasticity of tendons are related to the structured collagen matrix comprising the tendon (Wang et al., 2012). Similarly, the layering of collagen establishes transparency in the cornea (Espana and Birk, 2020). Additionally, matrix elements of the heart, in combination with the cytoskeleton of the myocytes, contribute to the passive mechanics of the heart (Granzier and Irving, 1995). Much of the ECM-based structural features of soft tissues involve classes of collagen, elastin, and proteoglycans (Holzapfel, 2000). These molecules establish the viscoelastic properties of the matrix environment and often are organized differently depending on the tissue type (Holzapfel, 2000; Ricard-Blum, 2011). For example, as mentioned earlier, tendons and ligaments exhibit highly aligned, thick collagen fibers that optimize the strength along one axis of the tissue. In contrast, interstitial tissues, which are less mechanically

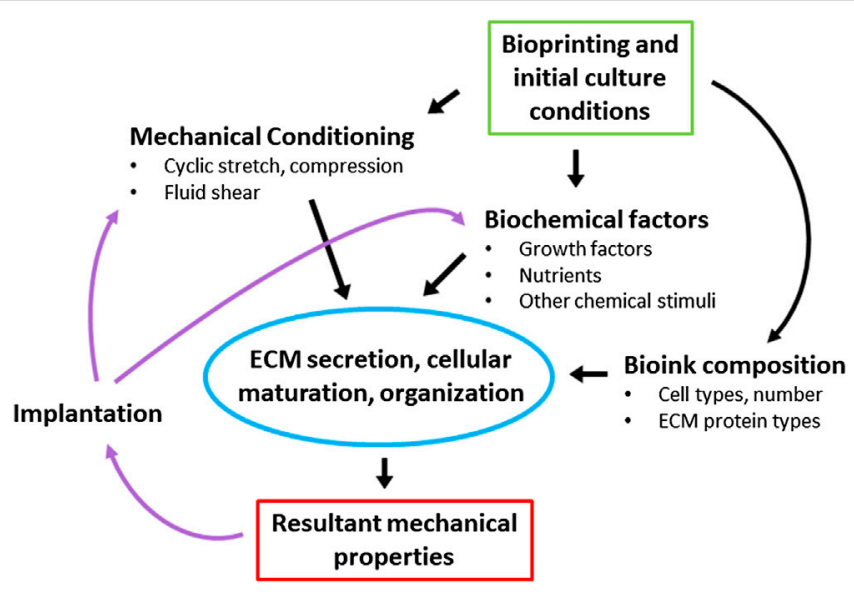

Figure 1 | The dynamics of tissue maturation. Initial determination of bioink composition, mechanical stimulation parameters, and biochemical stimuli will direct tissue remodeling and the resultant mechanical and functional properties of the tissue. This dynamic process continues after implantation, as the biological environment (mechanical and biochemical) triggers further remodeling and changes in tissue properties. 
robust, exhibit a loosely structured, porous net of matrix fibrils enabling nutrient delivery and diffusion (Jansen et al., 2018). Harder tissues, namely bone, have an additional large mineral component. About $70 \%$ of bone is comprised of the mineral hydroxyapatite. This gives the tissue great compression strength, while collagen (approximately 22\%) increases the tension and torsional strength of the tissue. Importantly, in the context of tissue fabrication, and bioprinting in particular, how much and of what type of matrix to be included in the print are essential for desired biological outcomes. The presence of one cue in the absence of another, while still resulting in a tissue being formed with some level of function, can lead to an outcome that is undesired (i.e., does not accurately recapitulate the in vivo setting). For example, while hydroxyapatite imparts a necessary stiffness, an excess can cause brittleness in bone (Zioupos et al., 1999; Augat and Schorlemmer, 2006). Similarly, different imbalances in the "matrisome" of the liver are thought to contribute to the variety of different liver diseases (Arteel and Naba, 2020). Breast cancer cells grown on matrices of similar stiffness yet providing different matrix-derived biochemical cues displayed different morphologies, differentiation states, and metabolic profiles (Ruud et al., 2020). Thus, a key challenging aspect of tissue fabrication and bioprinting is packaging enough matrix mechanics and biological signals in a form that is printable and amenable to manipulation. Fortunately, due to the highly dynamic nature of cell systems, all aspects do not necessarily need to be in place at the time of fabrication; only those features that can initiate and facilitate the evolution/maturation of the tissue once printed are required.

\section{THE TISSUE ENVIRONMENT: TISSUE MECHANICS}

As mentioned, most printing of tissue models and tissues involve one or more "post-processing" phases after the print. During this period, cells may proliferate to expand their numbers, secrete and organize matrix proteins to change the structural and mechanical properties of the tissue, and further differentiate toward more functional phenotypes. The inclusion of biochemical signals in the form of small molecule drugs, growth factors, or other media additives are common approaches to promote or guide post-print outcomes. Tissue mechanics can also be leveraged to influence cell biology and directly establish tissue function. For example, when mesenchymal stem cells are cultured in 3D gels, stiffer environments promote expression of bone differentiation markers, while softer environments promote myogenic and neural markers (Engler et al., 2006; Pek et al., 2010). Increased collagen fibril density can slow neovessel growth and reduce branching in a neovascular network formed by angiogenesis (Edgar et al., 2014b). Moreover, cells seeded in matrix with aligned elements will elongate and migrate faster, via contact guidance, than cells seeded in a disorganized matrix (Carey et al., 2016). Similarly, collagen fibrils patterned via applied mechanical strain orient mammary epithelium directed duct branching morphogenesis (Brownfield et al., 2013). Moreover, fibril organization affects cell migration, gene expression, matrix production and cell morphology in a variety of cell types (Wang et al., 2015; Newman et al., 2016). Cyclic compression of fabricated bone precursors promote osteogenesis and mineralization (Ravichandran et al., 2017). Conversely, cyclic stretch of engineered skeletal muscle improved elasticity and contraction strength (Powell et al., 2002; Moon du et al., 2008). In an angiogenic tissue environment, altering the boundary conditions of the environment such that growing neovessels generate anisotropic strains results in a neovasculature with parallel vessel segments (Chang et al., 2012; Underwood et al., 2014). However, intravascular blood flow abolished this aligned organization, which could be re-established if new boundary conditions were imposed during flow-induced remodeling (Chang et al., 2012). These examples highlight the concept that matrix composition and density, matrix microstructure, and boundary conditions, all profoundly influence stress-strain dynamics and therefore cell behavior and provide a means to manipulate the tissue environment to obtain desired functional outcomes after tissue fabrication.

\section{BIOPRINTING AND MECHANICAL MODELING}

Given that bioprinting is distinctly capable of rapidly creating structures with unique shapes, it is an ideal fabrication approach to incorporate the influence of boundary conditions in a tissue fabrication strategy. In addition to forming the tissue construct to specific anatomical shapes and features, bioprinting enables the inclusion of other features that, after externally and/or internally applied stresses or stimuli, leads to specific " $4 \mathrm{D}$ printed" outcomes. To fully leverage these approaches, computational modeling of tissue forces and movements will prove immensely useful in both designing initial printed shapes and predicting post-printing outcomes as the biology matures and remodels. For example, 4D printing approaches in combination with mathematical modeling of growth and morphogenesis are being explored in building brain tissue models and phantoms to study the effects of cortical folding in the context of stem cell behavior (Bayly et al., 2014; Esworthy et al., 2019). Similarly, computational studies of tissue growth or cellular fusion postprinting, dynamics of shear stresses in tissues, mass transfers, architectural features, etc. in the simulation of conformational changes and the influence of boundary conditions will have a significant impact on tissue fabrication. Specific to bioprinting, mechanobiological studies are contributing to the development of bioprinting materials with tunable mechanical and rheological properties (Vijayavenkataraman et al., 2018). Additionally, decades of work examining biomechanical processes, too many to cite here, is relevant to bioprinting-based strategies for tissue fabrication. Modeling of collagen fibril dispersion within soft tissues to predict stress and deformation characteristics (Holzapfel et al., 2019) coupled with finite element modelbased simulations of growing neovessel and collagen fibril dynamics during angiogenesis, (Edgar et al., 2014a; Edgar et al., 2014b; Edgar et al., 2015), for example, could be useful in designing final microvascular topologies. An understanding of the cellular aggregate fusion process and tissue environment 
dynamics could provide a design framework to fabricate tissue microarchitectures that are beyond bioprinting resolution (Sun et al., 2014; Robu et al., 2019). In a different application, but still relevant to bioprinting, computational force modeling of the tip movement inside gels with different viscoelastic properties was used to design and create channels within the material (Barua et al., 2020). Similarly, a combination of physical modeling of acoustic waves and engineering solutions was used to pattern cells within a bioprinted cartilage precursor via ultrasound (Chansoria et al., 2019).

\section{EVALUATING ENGINEERED TISSUE MECHANICS}

The mechanical properties of bioprinted tissues are assessed as with any other tissue, native or constructed. As such, choosing which tests to perform, and how to perform them, is often as important as the test itself. For example, bone, which primarily experiences compressive forces, typically undergoes compressive testing, in contrast to skin or ligaments which are assessed for tensile strength. Importantly, most tissues are anisotropic and exhibit non-linear stress-strain dynamics, which results in different elastic moduli depending on the magnitude and frequency of the applied stresses (Holzapfel and Ogden, 2003). Thus, it is important when performing such measurements that physiologically relevant stresses are applied. In printing vascular grafts, matching the native elastic modulus is critical for graft success. If the graft is too stiff or too elastic it can create complications that lead to stenosis, failure, and potentially death (Abbott et al., 1987). This can be challenging, as mechanical properties vary from person to person and between healthy and diseased tissue. If the modulus is measured at stresses much higher or much lower than what a vessel would normally experience, it will not accurately predict vessel behavior in vivo. Thus, measuring the elastic modulus properly, at physiological stresses, is essential for obtaining an accurate tissue assessment (Holzapfel and Ogden, 2003). Here, $3 \mathrm{D}$ printing can be advantageous as structures facilitating testing can be incorporated into the tissue design. Tensile testing typically involves holding two points of a tissue and stretching it, either cyclically or until failure. Inclusion of grip tabs as part of the tissue to facilitate tissue clamping or gripping affords easier use in the testing process and potentially mitigates complications related to gluing or joining grip tabs (Strobel et al., 2018).

Indentation testing, where a small probe applies force to a tissue and records force and displacement, is being used more as a non-destructive method to determine the elastic modulus of a tissue under non-destructive stresses (Samur et al., 2007). This method can yield different results than standard tensile tests, although as long as appropriate controls are used the data is equally valid (McKee et al., 2011). In the future, perhaps nondestructive testing methods can be incorporated directly within tissue bioreactors. This would enable better monitoring of tissue mechanics during maturation, and protocols such as biochemical and mechanical stimulation could be automatically modified to fine-tune the mechanical properties of the construct.
An important aspect of fabricating tissues, particularly for therapeutic use, relates to the usability by the clinical end user. For example, if a fabricated tissue graft is to be sewn in, then issues of suture retention and mechanical matching to the tissues at the site of implant become design parameters. Similarly, "surgical feel" and "handleability" of the tissue implant, such that it withstands the manipulations involved in implanting and fixating the tissue into the patient, are also important to explore. While bone tissues, by their nature, are rigid and sufficiently robust to be handled, their ability to accommodate fixation screws and plates may need to be considered. Thus, not only the tissue application but also how it's implemented, whether as a clinical implant or as a tissue model, is important to consider in the design and fabrication process.

\section{SUMMARY AND PERSPECTIVES}

Whether the outcome is to support weight loading, retain sutures, or resist shear, for example, many tissues are designed and fabricated to meet select mechanical specifications in a variety of applications. Furthermore, the tissue products may need to be uniquely shaped or constructed to reflect its use, such as a bone graft fitted to the geometry of a segmental defect or a tissue model configured to accommodate an assay format. As such, bioprinting is an enabling approach to fabricate bespoke tissue forms while preserving much of the biology relevant to forming tissues. Numerous factors contribute to the final fabrication outcomes, including the mechanical properties of any materials used in printing the initial tissue construct, but none more important than the dynamic interplay between the cellular elements of the tissue construct and the surrounding tissue environment. Within a tissue, native or fabricated, the cells exert force on (often as tension) and change the tissue environment while the environmental mechanics influence cell behavior in a complex reciprocal manner. Indeed, the responsiveness and adaptability that occurs during and after tissue fabrication is a hallmark features of biological systems. This dynamic may prove challenging in producing a prescribed tissue, as it can be important to match environmental cues to establish the desired tissue biology. However, it can also be leveraged in a bioprinting-based fabrication strategy to generate outcomes that might be otherwise difficult to establish. For example, some strategies use the internal forces generated by cells and cell systems in combination with initial boundary conditions, something readily established with bioprinting, to influence final tissue architecture and behavior. Others are building computational models to make predictions as to how a tissue form might change following cell activity or tissue construct maturation. There is considerable opportunity for innovative approaches that recognize the tissue will change, in response to the cues present, and designs a fabrication strategy that necessarily rely on cell behaviors responding to controllable mechanical cues.

While this perspective can be applied to many different applications involving fabricated tissues, it may not always be an option. The idea of manufacturing tissues "on demand," for 
example, would require constructs to be immediately implantable for patients who cannot afford to wait weeks and months for a tissue to mature. In this case, the same governing principles apply. However, the focus is on assembling the most appropriate combinations of materials, matrices, cellular elements, and design features that establish and maintain the necessary mechanical support needed for immediate implantation. Yet, it's important to consider that once implanted, the tissue construct will undergo changes, desired or otherwise, as dictated by the new tissue environment.

\section{REFERENCES}

Abaci, A., and Guvendiren, M. (2020). Designing decellularized extracellular matrix-based bioinks for 3D bioprinting. Adv. Healthc. Mater., 2000734. doi:10.1002/adhm.202000734

Abbott, W. M., Megerman, J., Hasson, J. E., L'Italien, G., and Warnock, D. F. (1987). Effect of compliance mismatch on vascular graft patency. J. Vasc. Surg. 5 (2), 376-382. doi:10.1067/mva.1987.avs0050376

Arteel, G. E., and Naba, A. (2020). The liver matrisome - looking beyond collagens. JHEP Rep. 2 (4), 100115. doi:10.1016/j.jhepr.2020.100115

Augat, P., and Schorlemmer, S. (2006). The role of cortical bone and its microstructure in bone strength. Age Ageing 35 (Suppl. 2), ii27-ii31. doi:10. 1093/ageing/afl081

Barua, R., Giria, H., Datta, S., Roy Chowdhury, A., and Datta, P. (2020). Force modeling to develop a novel method for fabrication of hollow channels inside a gel structure. Proc. Inst. Mech. Eng. H 234 (2), 223-231. doi:10.1177/ 0954411919891654

Bayly, P. V., Taber, L. A., and Kroenke, C. D. (2014). Mechanical forces in cerebral cortical folding: a review of measurements and models. J. Mech. Behav. Biomed. Mater. 29, 568-581. doi:10.1016/j.jmbbm.2013.02.018

Bedell, M. L., Navara, A. M., Du, Y., Zhang, S., and Mikos, A. G. (2020). Polymeric systems for bioprinting. Chem. Rev., 120 (19), 10744-10792. doi:10.1021/acs. chemrev.9b00834

Brownfield, D. G., Venugopalan, G., Lo, A., Mori, H., Tanner, K., Fletcher, D. A., et al. (2013). Patterned collagen fibers orient branching mammary epithelium through distinct signaling modules. Curr. Biol. 23 (8), 703-709. doi:10.1016/j. cub.2013.03.032

Carey, S. P., Goldblatt, Z. E., Martin, K. E., Romero, B., Williams, R. M., and Reinhart-King, C. A. (2016). Local extracellular matrix alignment directs cellular protrusion dynamics and migration through Racl and FAK. Integr. Biol. (Camb) 8 (8), 821-835. doi:10.1039/c6ib00030d

Castilho, M., de Ruijter, M., Beirne, S., Villette, C. C., Ito, K., Wallace, G. G., et al. (2020). Multitechnology biofabrication: a new approach for the manufacturing of functional tissue structures? Trends. Biotechnol. [Epub ahead of print]. doi:10.1016/j.tibtech.2020.04.014

Chan, W. W., Yeo, D. C. L., Tan, V., Singh, S., Choudhury, D., and Naing, M. W. (2020). Additive biomanufacturing with collagen inks. Bioengineering (Basel) 7 (3), E66. doi:10.3390/bioengineering7030066

Chang, C. C., Krishnan, L., Nunes, S. S., Church, K. H., Edgar, L. T., Boland, E. D., et al. (2012). Determinants of microvascular network topologies in implanted neovasculatures. Arterioscler Thromb. Vasc. Biol. 32 (1), 5-14. doi:10.1161/ atvbaha.111.238725

Chansoria, P., Narayanan, L. K., Schuchard, K., and Shirwaiker, R. (2019). Ultrasound-assisted biofabrication and bioprinting of preferentially aligned three-dimensional cellular constructs. Biofabrication 11 (3), 035015. doi:10. 1088/1758-5090/ab15cf

Chimene, D., Lennox, K. K., Kaunas, R. R., and Gaharwar, A. K. (2016). Advanced bioinks for 3D printing: a materials science perspective. Ann. Biomed. Eng. 44 (6), 2090-2102. doi:10.1007/s10439-016-1638-y

Clegg, J. R., Wagner, A. M., Shin, S. R., Hassan, S., Khademhosseini, A., and Peppas, N. A. (2019). Modular fabrication of intelligent material-tissue interfaces for bioinspired and biomimetic devices. Prog. Mater. Sci. 106, 100589. doi:10.1016/ j.pmatsci.2019.100589

\section{AUTHOR CONTRIBUTIONS}

The article was written, edited, and approved by HS, SM, and JH.

\section{FUNDING}

Supported by NHLBI of the National Institutes of Health under award number R01HL131856 and Department of Defense (W911NF-17-3-003/T0055).

Cui, X., Li, J., Hartanto, Y., Durham, M., Tang, J., Zhang, H., et al. (2020). Advances in extrusion 3D bioprinting: a focus on multicomponent hydrogel-based bioinks. Adv. Healthc. Mater 9 (15), 1901648. doi:10.1002/adhm.201901648

Datta, P., Ayan, B., and Ozbolat, I. T. (2017). Bioprinting for vascular and vascularized tissue biofabrication. Acta Biomater 51, 1-20. doi:10.1016/j. actbio.2017.01.035

Discher, D. E., Mooney, D. J., and Zandstra, P. W. (2009). Growth factors, matrices, and forces combine and control stem cells. Science 324 (5935), 1673-1677. doi:10.1126/science. 1171643

Edgar, L. T., Hoying, J. B., Utzinger, U., Underwood, C. J., Krishnan, L., Baggett, B. K., et al. (2014a). Mechanical interaction of angiogenic microvessels with the extracellular matrix. J. Biomech. Eng. 136 (2), 021001. doi:10.1115/1.4026471

Edgar, L. T., Hoying, J. B., and Weiss, J. A. (2015). In silico investigation of angiogenesis with growth and stress generation coupled to local extracellular matrix density. Ann. Biomed. Eng. 43 (7), 1531-1542. doi:10.1007/s10439-0151334-3

Edgar, L. T., Underwood, C. J., Guilkey, J. E., Hoying, J. B., and Weiss, J. A. (2014b). Extracellular matrix density regulates the rate of neovessel growth and branching in sprouting angiogenesis. PloS One 9 (1), e85178. doi:10.1371/ journal.pone.0085178

Engler, A. J., Sen, S., Sweeney, H. L., and Discher, D. E. (2006). Matrix elasticity directs stem cell lineage specification. Cell 126 (4), 677-689. doi:10.1016/j.cell. 2006.06.044

Espana, E. M., and Birk, D. E. (2020). Composition, structure and function of the corneal stroma. Exp. Eye Res. 198, 108137. doi:10.1016/j.exer.2020.108137

Esworthy, T. J., Miao, S., Lee, S.-J., Zhou, X., Cui, H., Zuo, Y. Y., et al. (2019). Advanced $4 \mathrm{D}$-bioprinting technologies for brain tissue modeling and study. Int. J. Soc. Netw. Min. 10 (3), 177-204. doi:10.1080/19475411. 2019.1631899

GhavamiNejad, A., Ashammakhi, N., Wu, X. Y., and Khademhosseini, A. (2020). Crosslinking strategies for 3D bioprinting of polymeric hydrogels. Small 16 (35). doi:10.1002/smll.202002931

Granzier, H. L., and Irving, T. C. (1995). Passive tension in cardiac muscle: contribution of collagen, titin, microtubules, and intermediate filaments. Biophys. J. 68 (3), 1027-1044. doi:10.1016/s0006-3495(95)80278-x

Holzapfel, G. A. (2000). "Biomechanics of soft tissue," in Handbook of material behavior. 1st Edn, Editors J. Lemaitre (Cambridge, MA: Academic Press), 7.

Holzapfel, G. A., and Ogden, R. W. (2003). Biomechanics of soft tissue in cardiovascular systems. Berlin, Germany: Springer Science \& Business Media.

Holzapfel, G. A., Ogden, R. W., and Sherifova, S. (2019). On fibre dispersion modelling of soft biological tissues: a review. Proc. Math. Phys. Eng. Sci. 475 (2224), 20180736. doi:10.1098/rspa.2018.0736

Jansen, K. A., Licup, A. J., Sharma, A., Rens, R., MacKintosh, F. C., and Koenderink, G. H. (2018). The role of network architecture in collagen mechanics. Biophys. J. 114 (11), 2665-2678. doi:10.1016/j.bpj.2018.04.043

Li, S., Liu, Y., Li, Y., Liu, C., Sun, Y., and Hu, Q. (2016). A novel method for fabricating engineered structures with branched micro-channel using hollow hydrogel fibers. Biomicrofluidics 10 (6), 064104. doi:10.1063/1.4967456

Mao, S., Pang, Y., Liu, T., Shao, Y., He, J., Yang, H., et al. (2020). Bioprinting of in vitro tumor models for personalized cancer treatment: a review. Biofabrication 12 (4), 042001. doi:10.1088/1758-5090/ab97c0

McKee, C. T., Last, J. A., Russell, P., and Murphy, C. J. (2011). Indentation versus tensile measurements of Young's modulus for soft biological tissues. Tissue Eng. B Rev. 17 (3), 155-164. doi:10.1089/ten.teb.2010.0520 
Moon du, G., Christ, G., Stitzel, J. D., Atala, A., and Yoo, J. J. (2008). Cyclic mechanical preconditioning improves engineered muscle contraction. Tissue Eng. 14 (4), 473-482. doi:10.1089/tea.2007.0104

Mota, C., Camarero-Espinosa, S., Baker, M. B., Wieringa, P., and Moroni, L. (2020). Bioprinting: from tissue and organ development to in Vitro models. Chem. Rev., 120 (19), 10547-10607. doi:10.1021/acs.chemrev.9b00789

Murphy, W. L., McDevitt, T. C., and Engler, A. J. (2014). Materials as stem cell regulators. Nat. Mater. 13 (6), 547-557. doi:10.1038/nmat3937

Nakanishi, Y., Okada, T., Takeuchi, N., Kozono, N., Senju, T., Nakayama, K., et al. (2019). Histological evaluation of tendon formation using a scaffold-free threedimensional-bioprinted construct of human dermal fibroblasts under in vitro static tensile culture. Regen Ther 11, 47-55. doi:10.1016/j.reth.2019.02.002

Newman, P., Galenano Niño, J. L., Graney, P., Razal, J. M., Minett, A. I., Ribas, J., et al. (2016). Relationship between nanotopographical alignment and stem cell fate with live imaging and shape analysis. Sci. Rep. 6, 37909. doi:10.1038/ srep37909

Pek, Y. S., Wan, A. C., and Ying, J. Y. (2010). The effect of matrix stiffness on mesenchymal stem cell differentiation in a $3 \mathrm{D}$ thixotropic gel. Biomaterials 31 (3), 385-391. doi:10.1016/j.biomaterials.2009.09.057

Powell, C. A., Smiley, B. L., Mills, J., and Vandenburgh, H. H. (2002). Mechanical stimulation improves tissue-engineered human skeletal muscle. Am. J. Physiol. Cell Physiol. 283, C1557-C1565. doi:10.1152/ajpcell.00595.2001

Ravichandran, A., Lim, J., Chong, M. S. K., Wen, F., Liu, Y., Pillay, Y. T., et al. (2017). In vitro cyclic compressive loads potentiate early osteogenic events in engineered bone tissue. J. Biomed. Mater. Res. B Appl. Biomater. 105 (8), 2366-2375. doi:10.1002/jbm.b.33772

Ricard-Blum, S. (2011). The collagen family. Cold. Spring. Harb. Perspect. Biol. 3 (1), a004978. doi:10.1101/cshperspect.a004978

Robu, A., Mironov, V., and Neagu, A. (2019). Using sacrificial cell spheroids for the bioprinting of perfusable $3 \mathrm{D}$ tissue and organ constructs: a computational study. Comput. Math. Methods Med. 2019, 7853586. doi:10.1155/2019/7853586

Ruud, K. F., Hiscox, W. C., Yu, I., Chen, R. K., and Li, W. (2020). Distinct phenotypes of cancer cells on tissue matrix gel. Breast Cancer Res. 22 (1), 82. doi:10.1186/s13058-020-01321-7

Samur, E., Sedef, M., Basdogan, C., Avtan, L., and Duzgun, O. (2007). A robotic indenter for minimally invasive measurement and characterization of soft tissue response. Med. Image Anal. 11 (4), 361-373. doi:10.1016/j.media.2007.04.001

Sasikumar, S., Chameettachal, S., Kingshott, P., Cromer, B., and Pati, F. (2020). 3D hepatic mimics - the need for a multicentric approach. Biomed. Mater. 15 (5), 052002. doi:10.1088/1748-605X/ab971c

Skylar-Scott, M. A., Uzel, S. G. M., Nam, L. L., Ahrens, J. H., Truby, R. L., Damaraju, S., et al. (2019). Biomanufacturing of organ-specific tissues with high cellular density and embedded vascular channels. Sci. Adv. 5 (9), eaaw2459. doi:10.1126/sciadv.aaw2459

Strobel, H. A., Calamari, E. L., Beliveau, A., Jain, A., and Rolle, M. W. (2018). Fabrication and characterization of electrospun polycaprolactone and gelatin composite cuffs for tissue engineered blood vessels. J. Bus. Manag. Res. Part B 106B (2), 817-826. doi:10.1002/jbm.b.33871

Sun, Y., Yang, X., and Wang, Q. (2014). In-silico analysis on biofabricating vascular networks using kinetic Monte Carlo simulations. Biofabrication 6 (1), 015008. doi:10.1088/1758-5082/6/1/015008

Unagolla, J. M., and Jayasuriya, A. C. (2020). Hydrogel-based 3D bioprinting: a comprehensive review on cell-laden hydrogels, bioink formulations, and future perspectives." Appl. Mater Today 18, 100479. doi:10.1016/j.apmt. 2019.100479

Underwood, C. J., Edgar, L. T., Hoying, J. B., and Weiss, J. A. (2014). Cell-generated traction forces and the resulting matrix deformation modulate microvascular alignment and growth during angiogenesis. Am. J. Physiol. Heart Circ. Physiol. 307 (2), H152-H164. doi:10.1152/ajpheart.00995.2013

Vijayavenkataraman, S., Yan, W. C., Lu, W. F., Wang, C. H., and Fuh, J. Y. H. (2018). 3D bioprinting of tissues and organs for regenerative medicine. $A d v$. Drug Deliv. Rev. 132, 296-332. doi:10.1016/j.addr.2018.07.004

Wang, J. H., Guo, Q., and Li, B. (2012). Tendon biomechanics and mechanobiology--a minireview of basic concepts and recent advancements. J. Hand Ther. 25 (2), 133-140; quiz 141. doi:10.1016/j.jht. 2011.07.004

Wang, S., Zhong, S., Lim, C. T., and Nie, H. (2015). Effects of fiber alignment on stem cells-fibrous scaffold interactions. J. Mater. Chem. B 3 (16), 3358-3366. doi:10.1039/c5tb00026b

Whitford, W. G., and Hoying, J. B. (2016). A bioink by any other name: terms, concepts and constructions related to 3D bioprinting. Future Science OA 2 (3), FSO133. doi:10.4155/fsoa-2016-0044

Wu, C., Wang, B., Zhang, C., Wysk, R. A., and Chen, Y. W. (2017). Bioprinting: an assessment based on manufacturing readiness levels. Crit. Rev. Biotechnol. 37 (3), 333-354. doi:10.3109/07388551.2016.1163321

Xing, F., Xiang, Z., Rommens, P. M., and Ritz, U. (2020). 3D bioprinting for vascularized tissue-engineered bone fabrication. Materials (Basel) 13 (10), 2278. doi: $10.3390 / \mathrm{ma} 13102278$

Zioupos, P., Currey, J. D., and Hamer, A. J. (1999). The role of collagen in the declining mechanical properties of aging human cortical bone. J. Biomed. Mater. Res. 45 (2), 108-116. doi:10.1002/(sici)1097-4636(199905)45:2<108::aid-jbm5>3.0.co;2-a

Conflict of Interest: JBH is a partner in Advanced Solutions Life Sciences. The remaining authors are employed at Advanced Solutions Life Sciences.

Copyright (c) 2020 Strobel, Moss and Hoying. This is an open-access article distributed under the terms of the Creative Commons Attribution License (CC $B Y)$. The use, distribution or reproduction in other forums is permitted, provided the original author(s) and the copyright owner(s) are credited and that the original publication in this journal is cited, in accordance with accepted academic practice. No use, distribution or reproduction is permitted which does not comply with these terms. 